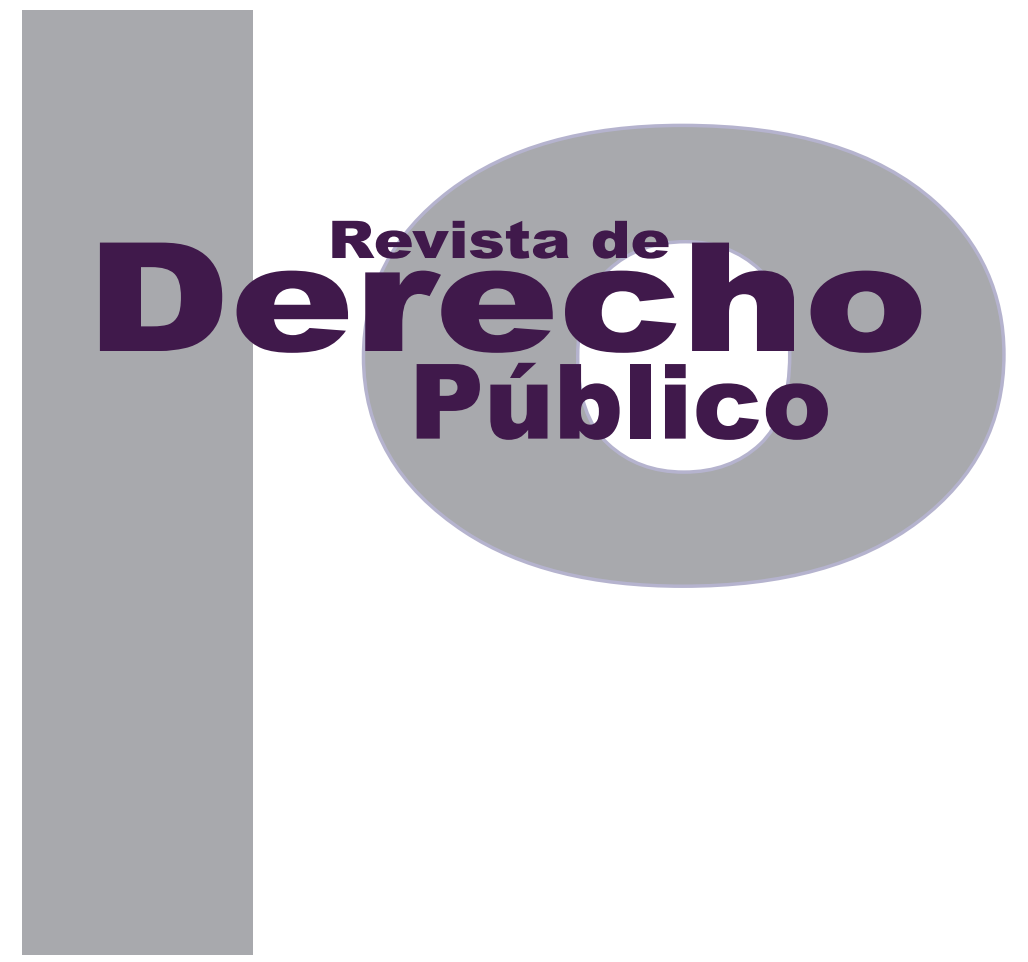

\title{
AlgunAS REFLEXIONES CON RESPECTO A LA ENSEÑANZA DEL DERECHO PROCESAL
}

Fernando Arrázola Jaramillo

Universidad de los Andes

Facultad de Derecho

Revista de Derecho Público N. ${ }^{\circ} 29$

Julio - Diciembre de 2012. ISSN 1909-7778 


\title{
Algunas reflexiones con respecto a la enseñanza del derecho procesal
}

\author{
Fernando Arrázola Jaramillo*
}

\begin{abstract}
RESUMEN
La enseñanza del derecho procesal no es tarea fácil. La complejidad en su pedagogía guarda relación con algunos aspectos tales como el bajo interés que despierta en los estudiantes, la elevada importancia que estos le asignan a dicha disciplina para su vida profesional, y la dificultad inherente a la comprensión y aplicación de algunos conceptos procesales. Por lo anterior, no todo el que entiende el derecho procesal está necesariamente dotado para enseñarlo. El presente escrito es un artículo de reflexión con respecto a la enseñanza del derecho procesal, fruto de una investigación realizada con algunos estudiantes de la Facultad de Derecho de la Universidad de los Andes de la ciudad de Bogotá, D.C., República de Colombia, durante el segundo semestre del año 2010, y busca brindar a los docentes de las disciplinas procesales algunas sugerencias y recomendaciones para hacer más amena y efectiva su enseñanza.
\end{abstract}

Palabras clave: enseñanza, derecho, derecho procesal, percepciones, estudiantes, docentes, características, metodologías, recomendaciones.
The teaching of procedural law is no easy task. The complexity of its teaching is related to certain aspects such as the scarce interest students show towards it, the importance they assign to such discipline for their professional lives, and the inherent difficulty in the comprehension and implementation of certain procedural concepts. Therefore, not everyone who understands procedural law is qualified to teach it. This article reflects on the teaching of procedural law, as a result of an investigation that was carried out with some law students of the Faculty of Law of the Universidad de los Andes in Bogotá, D.C., Colombia, during the second semester of 2010 , and it intends to provide procedural law teachers with some suggestions and recommendations in order to make its teaching more pleasant and effective.

KEY WORDS: teaching, law, procedural law, perceptions, students, teachers, characteristics, methodologies, recommendations. 


\section{SUMARIO}

Introducción - I. PERCEPCIONES DE LOS ESTUDIANTES CON RESPECTO AL DERECHO PROCESAL - $A$. Interés en el derecho procesal - B. Importancia del derecho procesal - II. PREPARACIÓN EN DERECHO PROCESAL - III. CARACTERÍSTICAS DE LOS DOCENTES DE DERECHO - A. Pasión por la enseñanza - $B$. Paciencia - C. Habilidades de comunicación - D. Manejo y trato personal del alumno-E. Innovación - F. Conocimiento de la teoría y de la práctica - G. Relacionar conceptos con la realidad - IV. ENSEÑANZA DEL DERECHO EN GENERAL - V. ENSEÑANZA DEL DERECHO PROCESAL - A. El aprendizaje basado en problemas (ABP) - B. Los juicios simulados - C. El método de casos - D. La asistencia a juzgados, oficinas de abogados y oficinas estatales - E. La cátedra magistral - F. Otras alternativas pedagógicas - VI. CONCLUSIONES - Bibliografía. 


\section{Introducción}

El entorno jurídico colombiano ha sido tradicionalmente litigioso. Consideramos un hecho notorio el que los despachos judiciales del país se encuentran literalmente abarrotados de procesos aún sin fallar. La congestión judicial es un problema que definitivamente no ha podido resolver el Estado colombiano. Muchos intentos de reforma judicial se han hecho con el fin de solucionar este delicado problema, siendo los más recientes de ellos la Ley 794 de 2003, la Ley 906 de 2004, la Ley 1395 de 2010 y la Ley 1564 de 2012 o Código General del Proceso. Sin embargo, la congestión judicial parece no ceder terreno.

No obstante lo anterior, resulta paradójico que la congestión judicial ponga de presente cuán importante resulta el derecho procesal para un abogado que pretenda ejercer su oficio en CoIombia. En algún momento de su carrera profesional, un abogado se verá enfrentado a un proceso judicial. Por alguna razón que no es objeto de este escrito, parece ser que los colombianos preferimos someter primero los problemas al conocimiento de la jurisdicción estatal en lugar de intentar resolverlos previamente por cualquiera de los mecanismos alternativos que la misma ley contempla. Así, como la labor del abogado es procurar una solución a los problemas de sus clientes, muchos de ellos solo encontrarán respuesta a través de los procedimientos, cuya enseñanza sí es objeto de este escrito.

El derecho procesal no es una asignatura cualquiera. Luego de más de veinte años de ejercicio profesional y de actividad docente universitaria, es posible evidenciar que hay algo curioso y especial alrededor de su enseñanza, pues siendo esta una disciplina instrumental cuya finalidad consiste precisamente en hacer efectivos los derechos sustanciales reconocidos en el ordenamiento jurídico, parece resultar más compleja su comprensión y asimilación por parte de los estudiantes que la de los mismos derechos cuya efectividad justamente busca garantizar el derecho procesal.

Tal vez lo anterior se explique por la dispersión de las normas y el elevado número de ellas, por las deficiencias de índole legislativa, por la dificultad que entraña su armonización e interpretación, por las excesivas reformas a los procedimientos o, simplemente, por lo engorroso de los procedimientos judiciales, lo que a la vez convierte su enseñanza en un verdadero reto que no todos los docentes terminan superando. Varios han sido los profesores que, siendo altamente calificados desde el punto de vista profesional, naufragan irremediablemente en las aguas turbulentas de la enseñanza del derecho procesal.

Estas dificultades en la pedagogía del derecho procesal fueron precisamente las que motivaron la investigación que llevó a cabo el autor de este escrito, durante el segundo semestre del año 2010, con los estudiantes y docentes de dos de las tres secciones del curso Argumentación en Procesos Civiles, de una sección de la clase de Procedimientos, y con algunos alumnos del Consultorio Jurídico, todos de la Facultad de Derecho de la Universidad de los Andes, ubicada en la ciudad de Bogotá, República de Colombia. 
El propósito de este artículo académico es, por ende, reflexionar con respecto a los resultados obtenidos en la referida investigación, plantear algunas ideas y formular algunas recomendaciones que puedan ser implementadas en el salón de clase, por parte de los docentes del derecho procesal.

\section{PERCEPCIONES DE LOS ESTUDIANTES CON RESPECTO AL DERECHO PROCESAL}

Para comenzar, consideramos que hay una información de vital importancia para un docente de cualquier asignatura, pero tal vez más cuando se trata del derecho procesal, y es aquella que se relaciona con el estado de ánimo de los estudiantes con respecto a la temática que va a ser objeto de estudio, es decir, cuánto interés o cuánto gusto manifiestan los estudiantes por el tema y qué importancia consideran que este puede tener en su vida profesional.

Conocer anticipadamente esta percepción puede ser determinante desde el punto de vista pedagógico, pues la manera como se aborden los distintos temas; las estrategias pedagógicas que se utilicen, tanto dentro como fuera del salón de clase; y de qué manera se puede buscar la motivación, el estudio y la participación de los estudiantes, pueden variar de manera significativa cuando se tiene esa información de antemano.

No resulta sencillo hacer generalizaciones acerca de la percepción que tienen los estudiantes del derecho procesal, puesto que cada alumno tendrá una visión diferente y está bien que así sea. Sin embargo, puede decirse que parecen mayoría aquellos alumnos que perciben el derecho procesal como algo difícil de estudiar y asimilar, pero especialmente complejo de poner en práctica.

Esta dificultad que supone el estudio del derecho procesal ha sido reconocida por varios autores. Schneider (1987), por ejemplo, reconoce que para los estudiantes de derecho en los Estados Unidos el curso de Procedimiento Civil es probablemente el más complicado del primer año de estudios, pero resalta a su vez la importancia de este cuando afirma que los estudiantes no pueden realmente entender las demás asignaturas de ese primer año si no comprenden a la vez las instituciones del derecho procesal civil.

Por su parte, Teply \& Whitten (2003) explican por qué razón la materia de Procedimiento Civil es tan ajena a los intereses de los estudiantes de derecho y por qué resulta a veces tan compleja para ellos, y citan entre otras razones la deficiente redacción de las normas, el contexto histórico en el que estas fueron aprobadas y las vivencias personales de los estudiantes de derecho, entre otras.

La experiencia docente en el campo del derecho procesal confirma esa percepción de complejidad que mencionan los anteriores autores, idea que puede complementarse mencionando que a los estudiantes no parece representarles tanta dificultad el entender los conceptos teóricos de la disciplina, pero al momento de aplicar a un caso concreto esos mismos conceptos que creían debidamente entendidos y asimilados, viene el choque con la realidad. Es precisamen- 
te en esa coyuntura del proceso de aprendizaje que el estudiante descubre las dudas y los vacíos conceptuales que no pudo identificar durante el manejo teórico del concepto, y le cuesta trabajo superarlos. Tal vez esta situación sea parte de la causa de la percepción de dificultad que entraña el derecho procesal.

Esta imagen de la que hablábamos en líneas anteriores, es decir, la del derecho procesal como una disciplina compleja, puede complementarse con otra idea que también existe, en el sentido de que su estudio resulta de capital importancia para la vida profesional de cualquier abogado. Esa importancia que se le reconoce al derecho procesal genera a su vez interés por el mismo, y ese interés motiva a los estudiantes. El aprendizaje sin motivación puede tener un nivel bastante superficial, un nivel de comprensión bastante elemental y poca recordación a mediano y largo plazo; el aprendizaje con motivación puede ser profundo y durar toda una vida.

Los autores también rescatan la importancia de la motivación. Tan (2008-2009) menciona algunos de los factores relacionados con el estudiante, que resultan decisivos como motivación para aprender, tales como creer que las clases son importantes y útiles para su futuro, estar interesado en el contenido de la materia, tener una personalidad ávida de conocimiento, creer que se es importante para sus instructores y compañeros, etc. Para este autor, la motivación resulta fundamental para que el aprendizaje pueda darse.

Szklarski (2011), por su parte, considera que el interés y el progreso académico son los principales elementos que inciden en la motivación de un estudiante, y en cualquiera de estas dos variables, la actitud que asuma el profesor con respecto a ellas puede afectar tanto el interés del alumno como su percepción de progreso.

Nótese cómo estos autores mencionan dos factores que resultan decisivos para la motivación de un estudiante: el interés y la importancia que se le asigne a una disciplina para la vida profesional. La investigación que se realizó en la Universidad de los Andes, con los alumnos de derecho procesal, parece confirmar plenamente que estos dos factores resultan vitales para que un estudiante se sienta motivado, como se verá a continuación.

\section{A. Interés en el derecho procesal}

En la investigación que es objeto de reflexión en este escrito, dos de las preguntas de una encuesta que se llevó a cabo entre los estudiantes hacían referencia concreta al interés o el gusto que mostraban estos por el derecho procesal en general. Los resultados, aunque quisiéramos que fueran mejores, son alentadores para el cuerpo docente y para los mismos estudiantes.

La primera de las preguntas hacía referencia al gusto que evidenciaban los estudiantes por el derecho procesal, pero no individualmente considerado sino en comparación con otras áreas del derecho. La pregunta tenía varias opciones de respuesta y el estudiante debía señalar todas aquellas que fueran de su interés. En el caso de esta pregunta, solamente el $28.6 \%$ de los encuestados escogió el derecho procesal como un área de estudio que despertaba su interés. Hasta este punto, el panorama no parece alentador. 
Sin embargo, la segunda pregunta que se hizo hacía referencia al interés o gusto por el derecho procesal individualmente considerado, no en comparación con otras áreas del derecho. Afortunadamente, los resultados fueron bien distintos a aquellos obtenidos en la primera pregunta, pues el $78.6 \%$ de los encuestados respondió que sí les gustaba el derecho procesal, arrojando un resultado de respuestas negativas de tan solo el $21.4 \%$.

En este punto el lector podrá estar confundido por los resultados antes señalados. No parece razonable que, preguntados los estudiantes por el gusto que les despierta el derecho procesal en comparación con otras áreas del derecho, solamente el $28.6 \%$ de los encuestados haya escogido el derecho procesal; y a renglón seguido, preguntados esos mismos estudiantes por el gusto o interés que les despierta el derecho procesal individualmente considerado y no en comparación con otras áreas del derecho, ese porcentaje de respuestas positivas haya aumentado al $78.6 \%$ del total.

Consideramos razonable concluir que a la mayoría de los estudiantes les gusta el derecho procesal, pero les gustan más otras áreas del derecho como el derecho internacional o el derecho privado, y esta concepción puede tener implicaciones profundas en el salón de clase.

La principal se refiere a la motivación de los estudiantes para dedicar tiempo y esfuerzo al estudio del derecho procesal, para la realización de ejercicios, trabajos, talleres y demás actividades. Todos los que hemos pasado por las aulas escolares y universitarias sabemos que el tiempo limitado de un alumno con varias asignaturas no se reparte de manera equitativa entre estas. Naturalmente, aquellos temas que despiertan el mayor interés tendrán una posición preponderante en la agenda, dejando un tiempo casi residual para aquellas materias que no resultan tan interesantes, a menos que una deficiente calificación acumulada durante el semestre sea la motivación sustituta para dedicar tiempo a un tema.

Las clases de derecho procesal están plagadas de anécdotas en las que los estudiantes reconocen no haber hecho las lecturas indicadas o no haber trabajado fuera de clase los ejercicios, talleres, trabajos o investigaciones previamente asignadas, por cuanto otras materias del pénsum les exigieron mayor tiempo de estudio. Resultado: los estudiantes escogieron trabajar otros temas que los motivaban más, sacrificando así las actividades académicas de índole procesal.

Así las cosas, si bien es cierto que resulta positivo el que a los estudiantes mayoritariamente les guste el derecho procesal, según los resultados de la investigación antes mencionada, no resulta tan halagüeño el que prefieran otras áreas del derecho, en la medida en que un menor interés los llevará a no dedicar el tiempo y el esfuerzo que resultan necesarios para un debido aprendizaje de las instituciones jurídico procesales.

Ahora bien, cabe preguntarse cuáles son las razones por las que los estudiantes muestran interés en el derecho procesal, así este no sea tan intenso como el interés por otras asignaturas del derecho. La referida investigación nos da 
igualmente la respuesta, y tiene que ver con la importancia que los estudiantes reconocen al derecho procesal como herramienta vital para el ejercicio exitoso de la profesión. Entienden así los alumnos que los procedimientos son el camino establecido por el legislador para la materialización de los derechos sustanciales, y resulta lógico que aquello despierte algún interés en el estudiante de leyes.

En síntesis, el estudiante de derecho parece tener un interés en el derecho procesal en la medida en que no puede concebirse un abogado en ejercicio totalmente deslindado de esta rama, pero hay otras materias que interesan más a los alumnos y la investigación realizada muestra una tendencia de estos a dedicar más tiempo a aquellas, lo cual puede tener un efecto adverso en el desarrollo de las clases de derecho procesal y, por ende, en su comprensión y aprendizaje. He ahí uno de los grandes retos para los docentes de procedimientos.

\section{B. Importancia del derecho procesal}

El interés que despierta el derecho procesal está estrecha e indisolublemente ligado, como se mostró anteriormente, a la importancia que le asigna el estudiante a esta área del derecho. De esta manera, la investigación realizada resulta totalmente consistente en este aspecto, pues los resultados indican, sin lugar a equívocos, que los estudiantes reconocen tanto la utilidad como la importancia del derecho procesal en sus planes académicos y profesionales.

En la encuesta a la que venimos refiriéndonos, se preguntó a los estudiantes qué importancia tenían para ellos los cursos de derecho procesal en comparación con los otros que ofrece el programa de derecho, y las respuestas fueron contundentes: el $57.1 \%$ de quienes respondieron dijeron que tenían мuсна importancia, el 40.5\% dijo que tenían una REGULAR importancia, y tan solo el $2.4 \%$ (un solo encuestado) dijo que tenían PocA importancia.

Por otro lado, al preguntarse qué planes tenían los estudiantes en su inmediato futuro profesional una vez se graduaran de la universidad, el $31 \%$ de quienes respondieron la encuesta manifestaron que planeaban ejercer profesionalmente como abogados litigantes.

Finalmente, al preguntarse a los mismos estudiantes si consideraban que iban a necesitar el derecho procesal en su vida profesional, el 83.3\% de los encuestados respondió que мuсно, el $16.7 \%$ dijo que Poco, y NINGUNo de los estudiantes manifestó que creía no necesitarlo jamás en su vida profesional.

Las anteriores cifras indiscutiblemente muestran que los estudiantes reconocen y entienden la importancia que va a tener el derecho procesal en su vida profesional. Si a lo anterior se agrega que todos y cada uno de los estudiantes entrevistados reconocieron, sin vacilaciones, que el derecho procesal era supremamente importante en la vida de cualquier abogado, queda clara entonces la percepción de los estudiantes en este sentido.

En síntesis, puede decirse otra vez que los estudiantes muestran interés por el derecho procesal, lo que es positivo, pero muestran más 
interés por otras áreas diferentes del derecho, lo que no es tan positivo. Y que ese interés está fundado, primordialmente, en la importancia que le reconocen al derecho procesal como elemento indispensable en sus vidas profesionales, lo que es positivo, pero que no está cimentado en un gusto desinteresado por el contenido de la materia, lo que no es tan positivo.

Esos son los resultados, y con ellos debe diseñar las estrategias pedagógicas el docente de derecho procesal.

\section{PREPARACIÓN EN DERECHO PROCESAL}

Otro aspecto que resulta de gran importancia para un docente, especialmente cuando está encargado de dictar cursos de profundización en temas de derecho procesal, es conocer cuál es el nivel de preparación que poseen los estudiantes luego de haber cursado las anteriores materias introductorias o generales que, normalmente, se encuentran a cargo de distintos docentes en una misma o en diferente institución educativa.

El nivel de preparación de los estudiantes puede determinar el mayor o menor ritmo de trabajo que debe seguirse con el grupo, los materiales que resultan necesarios para el estudio de los temas, los conceptos que deben trabajarse con más o menos tiempo, así como la naturaleza de los ejercicios o trabajos que se deben elaborar, entre otros. Si no hay un manejo básico de instituciones procesales por parte de los alumnos, que sirva de punto de partida al docente y con base en el cual puedan construirse las actividades pedagógicas del semestre, puede resultar muy difícil el desarrollo y resultado del curso.

Por lo anteriormente señalado, en la encuesta que se hizo a los estudiantes de derecho procesal de la Universidad de los Andes en el año 2010, investigación que en este escrito es objeto de reflexión, se preguntó cuál era la percepción que tenían en cuanto a su propia preparación en derecho procesal; sorpresivamente solo el 4.8\% de los encuestados manifestó sentirse mur BIEN preparados, mientras que el $47.6 \%$ dijo sentirse solo BIEN preparados, y el restante $47.6 \%$ manifestó sentirse entre REGULAR Y MAL preparados. Es decir, los resultados arrojaron que el grupo de estudiantes encuestados está repartido en dos grandes bloques, con exactamente el mismo porcentaje cada uno: el de los que manifiestan sentirse bien o muy bien preparados y el de quienes expresan sentirse regular, mal o muy mal preparados.

Ahora bien, cabe preguntarse cuáles son las causas de esa buena o mala preparación en derecho procesal. La investigación encontró que los estudiantes identificaron una serie de variables a las cuales le atribuyen, en mayor o menor grado, la responsabilidad por su nivel de preparación en temas de derecho procesal. Sin embargo, de todas las variables mencionadas, las dos que los estudiantes identifican como más relevantes hacen relación con el profesor de la materia y con el sistema de enseñanza que ese mismo profesor emplee durante la sesión de clase.

Es así como el 73.8\% de los encuestados, casi las tres cuartas $(3 / 4)$ partes del total, identificó 
al profesor y al sistema de enseñanza como los factores más importantes de todos aquellos que inciden en su preparación en derecho procesal. Sin embargo, hay otros factores que, si bien no tienen la relevancia de los dos mencionados anteriormente, son significativos y deben ser tenidos en cuenta, tales como los materiales del curso, el programa, el sistema de evaluación y el tamaño de la clase. Sin embargo, a juzgar por las respuestas de los estudiantes, ninguno de ellos tiene una importancia siquiera parecida a la que le reconocen al profesor y su metodología de enseñanza.

Los anteriores hallazgos nos invitan a profundas reflexiones como docentes de derecho procesal. Si, a juzgar por la investigación, esos son los dos factores más relevantes que pueden influir en la preparación de un estudiante, es precisamente a ellos a los que debe prestarse más atención al momento de diseñar el correspondiente espacio pedagógico. Es por esta razón que en el presente escrito abordamos también el tema de cuáles calidades o características debe tener un buen docente de derecho procesal, y cuáles metodologías pueden servir a sus propósitos pedagógicos en el salón de clase.

\section{CARACTERÍSTICAS DE LOS DOCENTES DE DERECHO}

Acabamos de mencionar cómo resulta supremamente importante para un docente de derecho procesal conocer de antemano la percepción que tienen los estudiantes del tema objeto de estudio. Ello, tal como se dijo, le permitirá diseñar las estrategias pedagógicas y enfocar- las de manera importante hacia la motivación del alumnado.

Sin embargo, tal vez resulte aún más importante para el proceso de aprendizaje que el docente reúna las características necesarias para desenvolverse con maestría en un ambiente pedagógico complejo como el que puede presentarse, y con frecuencia se presenta, en una clase de derecho procesal.

La importancia que tienen las calidades del docente en el aprendizaje de los estudiantes la mencionan Rivkin, Hanushek \& Kain (2005), pero afirman que el impacto no es muy grande y se presenta más entre los estudiantes de grados inferiores. Aunque resulta en parte discutible lo que afirman estos autores, agregaríamos que el impacto pedagógico puede ser más significativo en aquellos estudiantes que no tienen un nivel académico tan elevado en comparación con sus compañeros o en aquellos alumnos que no se encuentran motivados para el estudio de un tema en particular. En esos casos, el docente resulta ser un factor pedagógico determinante.

La investigación también intentó buscar bibliografía que se refiriera a las características que debe tener un buen docente de derecho, lo cual parecería aún más aplicable a quienes se dedican a la pedagogía del derecho procesal. Sin embargo, la revisión bibliográfica encontró algunos pocos escritos que hacían referencia tangencial a los atributos que serían deseables en los docentes en general, independientemente de la disciplina concreta de que se trate. 
Arnon \& Reichel (2007) por ejemplo, mencionan que un profesor ideal debe tener dos condiciones fundamentales: por un lado, el conocimiento de la materia que dicta, y por el otro lado una personalidad adecuada. De acuerdo con algunos estudios a los cuales hacen referencia, lo más importante para los estudiantes es que el profesor explique bien y que pueda guiarlos adecuadamente en la solución de los problemas. En otros estudios que también citan estos autores, aparece que lo más importante son algunos aspectos de la personalidad del profesor, tales como su paciencia, comprensión, habilidad para comunicarse con el estudiante, su modestia, informalidad, simplicidad y otros.

Wildman (1942) hace referencia a aquellos docentes que son recordados por sus estudiantes, y los describe como aquellos que tienen conocimiento y pasión por el tema de la clase, aquellos que se interesan no solamente por el tema sino también por sus estudiantes como personas, aquellos que se interesan por el mundo que los rodea y que pueden asociar los temas de la clase con ese mundo en el que viven, aquellos que están inspirados al enseñar.

La escasa bibliografía encontrada en relación con las características de los docentes del derecho nos lleva a pensar que este escrito reflexivo constituye una oportunidad para complementar un poco este tema, con fundamento en la experiencia docente propia y en las observaciones que se han hecho a otros docentes a lo largo de varios años de carrera universitaria.

La pregunta que surge, entonces, sería la siguiente: ¿Cuáles son las características que debe tener un buen docente de derecho, y especialmente un buen docente de derecho procesal? Con base en la investigación realizada y la experiencia adquirida, podemos generalizar y concretar la respuesta de la siguiente manera:

\section{A. Pasión por la enseñanza}

Todo docente debe sentir verdadera pasión por la noble tarea de educar nuevas generaciones. La pasión se transmite casi de manera inmediata. No todos los profesores tienen vocación pedagógica, y solamente debería consagrarse a la docencia quien se apasione por ese oficio. No resulta suficiente ser una reconocida autoridad o un connotado tratadista en un tema, pues si se carece de la vocación para transmitir con amor, paciencia y dedicación dicha experiencia y conocimientos, el impacto pedagógico sobre el alumno va a ser muy limitado. Un gran docente no solo debe conocer a fondo su disciplina, sino también amar profundamente el ejercicio de su enseñanza.

\section{B. Paciencia}

Todo pareciera indicar que solamente tras repetir y repetir hasta el cansancio un concepto o una idea los estudiantes comienzan verdaderamente a entenderlo. No es fácil expresarlo de una mejor manera, pues cuando se trata de algunos temas que pueden resultar complejos como el derecho procesal, la paciencia es una virtud fundamental de cualquier docente.

Son muchas las ocasiones en las que la ausencia de preguntas por parte del auditorio puede llevar al profesor a pensar que el tema ya está 
claro para los estudiantes. Nada más alejado de la realidad. A pesar de repetir una y otra vez los conceptos, de explicarlos de muy diversa manera, de hacer toda clase de ejercicios teóricos y prácticos para intentar mostrar cómo se aplican, el docente se encuentra frecuentemente con la frustración de que, pensándose superados, dichos conceptos aún no aparecen claros para una parte del alumnado. La única virtud que permite manejar este tipo de situaciones es, precisamente, la paciencia.

Una situación como la mencionada anteriormente deriva en que el profesor deberá hacer una pausa y replantear sus explicaciones tantas veces como sea necesario, repasar una y otra vez los conceptos que aún no estén claros, hacer una y otra vez ejercicios prácticos que ayuden a aclarar las dudas, y dedicar tiempo y esfuerzo para que no queden alumnos rezagados en la clase, conservando siempre el criterio para decidir cuándo es pertinente detener a todo el salón y cuándo resulta mejor continuar con la clase y dar un manejo individual a uno o a más alumnos en dificultades, de tal forma que el grupo no pierda la dinámica de aprendizaje si la duda planteada no es compartida por un número significativo de estudiantes.

El docente debe estar dispuesto a dedicar el tiempo necesario, aun por fuera de los horarios señalados para las sesiones de clase y para la atención de estudiantes, con el fin de atender cualquier duda o cualquier inquietud que pueda tener un alumno en relación con los temas que son objeto de estudio. Quien no tenga esta disposición, quien no tenga paciencia, es mejor que dedique su tiempo a asuntos bien distintos de la docencia.

\section{Habilidades de comunicación}

El docente debe estar en capacidad de explicar los temas de tal forma que la comunicación con los alumnos sea fluida, clara y permanente. Lo anterior significa utilizar un lenguaje elemental que esté al alcance de todo estudiante, buscar ejemplos claros y concisos, redactar casos y ejercicios pertinentes, y en general, lograr la atención del auditorio en forma espontánea, procurando que las ideas se transmitan de la manera más elemental posible. Se trata de que los alumnos entiendan, no de descrestarlos con un lenguaje rebuscado y denso que los va a perder desde el comienzo de la sesión.

\section{Manejo y trato personal del alumno}

Un buen docente debe estar pendiente del progreso de todos sus estudiantes, y ojalá lo esté de cada uno de sus alumnos en particular. Cuando un estudiante es consciente de que el docente conoce su nombre, que está pendiente de su progreso, que espera trabajo y esfuerzo de su parte, y que no es un simple código o un número más dentro de un grupo de seres humanos, se sentirá más comprometido con el curso y su motivación tendrá un nivel infinitamente más elevado.

Adicionalmente, el buen docente deberá poder dar un trato adecuado al estudiante frustrado, a aquel a quien se le dificulta la materia de manera especial, a aquel que siente saberlo todo, 
a aquel que se muestra rebelde y amenaza con sabotear el buen discurrir de la sesión de clase $y$, en general, manejar de buena manera las situaciones individuales que se presenten en la clase.

\section{E. Innovación}

Un buen profesor siempre debe estar en capacidad de innovar. Resulta fundamental que las clases no se tornen repetitivas, con la misma dinámica y las mismas actividades una y otra vez, de tal manera que el estudiante pierda el interés en asistir a ellas o simplemente deje de involucrarse activamente en los procesos pedagógicos que allí se desarrollan.

En todas las relaciones humanas la rutina provoca desastres. En el salón de clase, donde se manejan tantas y tan complejas relaciones interpersonales, no tiene por qué ser diferente. Así, aunque la presión de hacer una actividad diferente cada día puede resultar estresante para el docente, los efectos en los estudiantes son indudablemente positivos.

\section{F. Conocimiento de la teoría y de la prác- tica}

Un buen docente de derecho no solamente debe manejar la dogmática, los conceptos y los principios en los que se cimenta la asignatura, sino que también debe conocer cómo dichos conceptos y principios tienen aplicación práctica en la vida real. No existe una mejor escuela que la experiencia, y el ejercicio profesional enseña y forma profesionales de la mejor manera.
Salvo por el caso de las ciencias exactas, y todos tenemos claro que el derecho no lo es, existe una brecha importante entre la teoría y la realidad, entre el deber ser y el ser. Los abogados vivimos a diario la frustración de observar un universo jurídico que no funciona como lo establecen las normas.

En la medida en que es misión de las instituciones educativas formar profesionales para la vida, para que puedan desempeñarse exitosamente en un medio real en el que van a trabajar y vivir, el docente debe mostrar a sus alumnos la visión y la experiencia de cómo es que esos conceptos teóricos se materializan en la realidad de una u otra manera, pues de lo contrario estará faltando a una de sus obligaciones fundamentales como formador de nuevas generaciones.

\section{G. Relacionar conceptos con la realidad}

La experiencia docente y las mismas manifestaciones de los estudiantes durante la investigación realizada, indican de manera inequívoca que todas las metodologías y experiencias de aprendizaje que se pongan en práctica, tanto dentro como fuera del salón de clase, deben tener alguna relación con la realidad del alumno.

Lo anterior impone una responsabilidad que gravita en cabeza del profesor, pues en los ejemplos que presente, en los talleres que realice, en los casos que elabore, en los trabajos que asigne, y en cualquier otra actividad pedagógica que programe, el alumno deberá estar en capacidad de relacionar el tema y el objeto de la actividad con alguna situación real que haya vivenciado o 
conocido, o que por lo menos pueda sucederle. Esto se constituye, además, en un importante factor de motivación para el estudiante.

\section{ENSEÑANZA DEL DERECHO EN GENERAL}

Antes de adentrarnos en las particularidades que evidencia el derecho procesal desde el punto de vista pedagógico, parece conveniente detenernos primero en el tema de la enseñanza del derecho en general, pues muchas de las recomendaciones o estrategias aplicables al derecho en general lo son también para el derecho procesal.

Existe abundante literatura que se refiere al método de casos que por tanto tiempo se ha implementado exitosamente en la escuela de leyes de la Universidad de Harvard, y el cual parece seguir predominando en las escuelas de leyes norteamericanas, método que está encaminado fundamentalmente a entrenar al estudiante para razonar de manera lógica, crítica, al igual que para reconocer los hechos relevantes y los problemas que se encuentran inmersos en un caso jurídico particular. En otras palabras, lo que busca es formar a los estudiantes para que piensen como abogados.

Sin embargo, son varios los escritos que han venido cuestionando dicho método de enseñanza del derecho, entre otras cosas por cuanto efectivamente entrena al estudiante en unos aspectos fundamentales del ejercicio profesional, como es el razonamiento lógico y la interpretación y análisis de jurisprudencia, pero deja por fuera otros elementos también fundamentales de la profesión como son el manejo del cliente, el entorno legal en el que ha de desenvolverse, la elaboración de demandas, y otros, por lo cual varias voces han propuesto métodos alternativos de enseñanza que encuentran más eficaces, todos ellos con un enfoque más práctico, y buscando siempre que semejen el ejercicio real del derecho.

Shapiro (2000-2011) por ejemplo, hace una comparación entre la enseñanza del derecho utilizando el método de casos y el aprendizaje a través de problemas, efectuando una crítica al primero y tomando partido por el segundo, pues considera que el aprendizaje a través de problemas lleva al estudiante menos al estudio de una jurisprudencia o doctrina legal de manera teórica, y más al análisis de hechos reales que pueden constituir un problema jurídico.

Wheaton (1936-1937) por su parte, considera que la enseñanza del derecho debe simular en lo posible el mundo que van a enfrentar los estudiantes en el momento en que se gradúen de la universidad, declarándose un abanderado de la práctica para la enseñanza del derecho. Así, cree que el alumno debe gastar un menor tiempo en el estudio de textos y análisis de jurisprudencia de las altas cortes y, en su lugar, debe emplear más tiempo como practicante en una oficina de abogados o atendiendo juicios reales en los tribunales de justicia.

De la misma manera, Frank (1933) formula un ataque directo contra el método Langdell de enseñanza del derecho, impuesto en las universidades americanas por mucho tiempo, en virtud 
del cual el profesor es un académico con muy poca o ninguna experiencia práctica en la realidad, enfocando los esfuerzos pedagógicos al estudio teórico de las normas y la jurisprudencia. En cambio, sugiere que las escuelas de leyes se parezcan mucho a las oficinas de abogados, en las que el profesor esté activamente participando en la solución de los problemas reales de los cuales se ocupa el derecho, y teniendo a los alumnos como sus aprendices en todo este proceso.

En sentido contrario, Garciandía González \& Liébana Ortiz (2011, p. 275) consideran que no se pueden "convertir las enseñanzas jurídicas en una suerte de formación profesional, que surta al mercado de <<prácticos>> carentes de espíritu crítico y experiencia profesional verdadera... las Facultades de Derecho deben centrarse fundamentalmente en la instrucción teórica de los estudiantes, puesto que la experiencia profesional acabarán adquiriéndola en el foro".

Parece existir una tendencia cada vez más generalizada que propugna por un enfoque práctico para la enseñanza del derecho, de manera que las actividades pedagógicas que se pongan en práctica en las aulas semejen cada vez más la vida real de un abogado.

Igualmente, cada vez resulta más aceptado un enfoque pedagógico según el cual el estudiante debe ser quien asuma cada vez más la responsabilidad de su propio aprendizaje, aliviando la carga que en este sentido ha asumido tradicionalmente el docente. Esta tendencia recibió un empuje fundamental con la construcción del denominado Espacio Europeo de Educación Su- perior, y concretamente con la Declaración de Bolonia del año 1999; las implicaciones desde el punto de vista de las metodologías de enseñanza han sido profundas.

\section{ENSEÑANZA DEL DERECHO PROCESAL}

La tendencia más o menos reciente de enfatizar el componente práctico en la enseñanza del derecho no es ajena al derecho procesal. Muy por el contrario, resulta aún más pertinente y generalizado el llamado a estos docentes para que procedan de esa manera, de tal forma que las estrategias pedagógicas que implementen en la clase sean lo más parecidas a la realidad del ejercicio profesional de un abogado.

Sin embargo, compartimos plenamente las observaciones que, con carácter general, hacen Garciandía González \& Liébana Ortiz (2011, p. 279) cuando reflexionan sobre las metodologías activas de enseñanza y afirman que

Con carácter previo al comentario de cada una de las propuestas, cabe hacer hincapié en el hecho de que para que funcionen debe contarse con una adecuada participación activa de todos los estudiantes. Esta se configura como el primer motor de la clase, puesto que el profesor debe atenerse simplemente a dirigir y a encauzar las reflexiones del alumnado. Sin el concurso de voluntades de los estudiantes estas propuestas docentes pierden su razón de ser, puesto que los estudiantes deben implicarse hasta el punto de consultar bibliografía e información adicional a la suministrada inicialmente por el docente. 
No hay metodología activa de enseñanza posible sin la participación activa e interesada del estudiante, tanto dentro como fuera del salón de clase. Muchas de las estrategias pedagógicas que puede implementar el docente del derecho procesal se fundamentan en lecturas previas, en investigación y en otros tipos de labores del estudiante fuera de la clase, motivo por el cual si el alumno ha omitido complementar el trabajo que realiza el docente y continúa con una actitud pasiva con respecto a su propio aprendizaje, la eficacia de cualquier estrategia pedagógica práctica puede verse seriamente comprometida.

Si bien los autores consultados durante el curso de la investigación difieren en cuanto a cuáles de los métodos prácticos pueden resultar más adecuados que otros, no se encontraron autores que sugirieran un enfoque puramente teórico para la enseñanza del derecho procesal, lo cual en manera alguna quiere decir que algunas metodologías como la cátedra magistral hayan terminado en total desuso, como se explicará más adelante.

Algunos docentes defienden el método de casos, otros abogan por el aprendizaje basado en problemas, otros por fomentar la práctica de estudiantes en oficinas de abogados o como ayudantes $u$ observadores en los tribunales de justicia, al igual que por otras que más adelante mencionaremos. En fin, la investigación encontró un sinnúmero de sugerencias, todas de carácter práctico, para la enseñanza del derecho procesal.

Teply \& Whitten (2003) resaltan la importancia del estudio de casos para la enseñanza del derecho procesal y la práctica de llevar al estudiante a trabajar con problemas.

Subrin (1993-1994) también afirma que los estudiantes deben poder entender las instituciones procesales mediante su aplicación a casos concretos, y especialmente a casos de la vida real que a ellos puedan llegar a sucederles. Hay que trabajar con casos de verdad, asumir roles, defender ideas y atacar otras, todo bajo una óptica práctica.

Sheinkopf Hoffman (2003) propone enseñar el derecho procesal civil bajo una modalidad del aprendizaje basado en problemas, pero la autora aclara que con esta metodología no se puede cubrir un número elevado de temas sino más bien pocos, pero en contraprestación le permite al estudiante comprender la complejidad de los mismos.

Anderson \& Kirkwood (1987) sugieren incluir en los cursos de derecho procesal civil una experiencia real de litigio para los estudiantes. Estos docentes elaboran un caso tomado de la vida real y lo aplican en la clase, de manera que el estudiante entienda los hechos del caso, redacte una demanda, la conteste y, en general, haga lo que haría un abogado litigante en la vida real.

En resumen, puede observarse que la tendencia de los docentes de derecho es a la realización de ejercicios prácticos que simulen la vida real de un abogado, como mecanismo efectivo que puede ayudar en el proceso pedagógico de una materia de las características especiales que tiene el derecho procesal civil. 
Ahora bien, existen varias alternativas pedagógicas para la enseñanza del derecho procesal que pueden ser acogidas e implementadas por los docentes de esta materia en el salón de clase, con el fin de lograr que los conceptos, principios e instituciones jurídicas propias de esta disciplina puedan ser comprendidos a cabalidad por los estudiantes. Aunque no todas las que a continuación se mencionan tienen un carácter o una connotación práctica, bien empleadas pueden contribuir a una mejor enseñanza y asimilación del derecho procesal. ¿Cuáles son mejores para lograr estos objetivos? Solamente el docente estará en condiciones de decidir a cuál de ellas acude según las circunstancias se lo recomienden.

\section{A. El aprendizaje basado en problemas (ABP)}

El aprendizaje basado en problemas, como su nombre lo indica, es una metodología a través de la cual un estudiante es presentado con un caso diseñado por el profesor, que no tiene una única solución correcta, ojalá tomado o inspirado en la vida real, el cual debe ser abordado por aquel sin previo estudio o familiarización con los problemas o conceptos que va a encontrar en el caso que le es presentado.

Esta metodología en particular busca que el estudiante desarrolle su capacidad de pensar de manera crítica, que identifique los problemas y los conceptos fundamentales que se encuentran inmersos dentro de un caso, que identifique sus metas de aprendizaje, que desarrolle la habilidad para brindar soluciones creativas a los problemas y, especialmente, que aprenda a enseñarse a sí mismo. Además, tiene un beneficio adicional y es que incrementa la motivación de los alumnos.

Es esta una metodología que ha sido diseñada para grupos pequeños de estudiantes y, por ende, resultará supremamente difícil ponerla en práctica en grupos grandes donde su manejo y eficacia pedagógica se van a ver seriamente comprometidos.

En la modalidad que más hemos utilizado para trabajar el ABP, cada tema cuyo estudio se aborde mediante esta metodología debe superarse en tres pasos o etapas definidas: la primera, en la que el profesor entrega el caso a los estudiantes y estos deben revisarlo cuidadosamente para identificar los problemas jurídicos inherentes, las instituciones jurídicas relevantes y los objetivos de aprendizaje que deben alcanzar; la segunda, en la que los alumnos traen a clase el resultado del trabajo que han hecho fuera de ella, sesión que es aprovechada también para que los alumnos y el profesor intervengan en la resolución de las dudas y en la aclaración de conceptos que presenten dificultad; y la tercera, que es fundamentalmente una sesión de reflexión con respecto al aprendizaje obtenido.

Aunque esta metodología resulta altamente recomendable para utilizar cuando se trata de temas jurídicos, y los beneficios pedagógicos que se obtienen resultan evidentes, tiene un inconveniente y es el tiempo que tarda cada tema en evacuarse satisfactoriamente. En el mundo complejo de hoy, donde los grupos de estudiantes son cada vez mayores y el tiempo es un elemento cada vez más escaso, resulta 
muy complicado evacuar una buena parte de los temas de un programa académico a través de esta metodología.

Una alternativa que permite maximizar el ABP consiste en integrar varios temas o módulos del programa en un solo caso, de tal forma que la demora que pueda presentarse por el empleo de varias sesiones de clase se vea compensada por el avance cualitativo y cuantitativo en el aprendizaje del curso. Sin embargo, repito, la cantidad de temas que integran los programas, el cada vez más elevado número de estudiantes, y la corta duración de un semestre académico, dificultan su empleo como metodología permanente.

Finalmente, debe mencionarse algo que hemos evidenciado y consiste en que no todos los estudiantes valoran y desean trabajar con esta metodología. Al contrario de lo que sería deseable, en las clases de derecho procesal un número significativo de estudiantes (que no se ha medido de manera confiable) han sugerido abiertamente que no sea utilizada para la enseñanza del derecho procesal, mientras otros la favorecen y se sienten muy a gusto. Esta misma situación es recogida por Caballol Angelats cuando nos relata que al utilizar la metodología ABP,

La respuesta de los estudiantes ha sido heterogénea. No quiero silenciar la reacción adversa de una parte del alumnado. Muchos de ellos tienen tan asumida la metodología tradicional que niegan hasta el extremo la posibilidad de tomar ninguna iniciativa que no haya sido previamente indicada por el profesor. ¡Primero la teoría y después la práctica! Insisten contrariados... En la vertiente positiva, los estudiantes que siguen el curso, evidencian una mayor y (sic) autoestima. Todos, se sienten motivados, independientemente del nivel que consiguen. Intervienen y preguntan (...) El principal inconveniente que detectan es que les queda mucho por aprender. Es frecuente que en las encuestas indiquen que hay poco tiempo para trabajar todo el temario. Finalmente, no niegan que una clase magistral les puede resultar más cómoda. (2011, p. 145).

Consideramos que la mejor sugerencia consiste en introducir la metodología ABP solamente en algunos temas del programa, reservando los más complejos para otras que puedan resultar más eficientes.

\section{B. Los juicios simulados}

La metodología de los juicios simulados busca acercar a los estudiantes a la realidad que entraña la práctica del derecho, permitiéndoles involucrarse dentro de un caso jurídico que puede ser real o elaborado por el docente, mediante la representación de las partes y su participación simulada en el juicio, lo que les permitirá ejercer el rol de uno cualquiera de los sujetos procesales durante toda la duración del proceso.

Esta metodología presenta enormes ventajas y resulta altamente positiva desde el punto de vista pedagógico, pues le permite al estudiante enfrentar una situación problemática práctica, que seguramente ha sido Ilevada a la jurisdicción por parte de sus interesados en busca de una solución, y aprender a manejar un caso jurídico desde la perspectiva de uno de sus participantes, fomentando su capacidad de análisis crítico, su creatividad, la búsqueda de solucio- 
nes jurídicas, la anticipación de los argumentos de la contraparte, la redacción de escritos y memoriales jurídicos, además de estimular el desarrollo de habilidades tan importantes como la expresión oral y el manejo corporal que les será tan útil ahora que la oralidad parece imponerse como regla técnica del derecho procesal.

Es corriente que este tipo de metodologías sean empleadas solamente en el último año de estudios de derecho, pues solo en ese momento el alumno habrá adquirido las herramientas que resultan necesarias para desempeñarse con destreza y excelencia en el rol que le ha sido asignado.

Como toda metodología práctica, los juicios o tribunales simulados demandan mucho tiempo y esfuerzo, tanto dentro como fuera del salón de clase, y lo exigen tanto del docente como del estudiante. La estructuración de un buen caso, muchas veces representado en un expediente judicial, puede resultar compleja y demorada, pero los resultados en el aprendizaje bien merecen el esfuerzo empleado.

La experiencia nos muestra que este tipo de metodologías en derecho resultan más útiles para el desarrollo de competencias, destrezas y habilidades en el estudiante, que para el estudio y comprensión de los temas que puedan corresponder a una asignatura en particular, los cuales pueden y deben ser aprendidos mediante otras estrategias pedagógicas, y más bien puestos en práctica a través de los juicios simulados.

Así las cosas, esta herramienta puede resultar muy útil para motivar al estudiante y enseñar- le la aplicación práctica de los conceptos de la clase, siempre que se cuente con el tiempo que una buena preparación y ejecución demandan.

\section{El método de casos}

El método de casos ha sido utilizado de manera generalizada en las escuelas de leyes en los Estados Unidos de Norteamérica, y se centra fundamentalmente en el estudio por parte del alumno de unos fallos jurisprudenciales emblemáticos decididos por las altas cortes de justicia. Los casos son seleccionados por el profesor de la clase y no por el estudiante. Con ellos se pretende que el alumno pueda asimilar los conceptos jurídicos que se discuten en el caso y Ios cuales son objeto de decisión (The Princeton Review). Este método es, fundamentalmente, una manera de estudiar las leyes y principios dogmáticos del derecho, no a través de los libros de tratadistas o mediante el estudio de códigos que traen una recopilación de normas sustantivas, sino a través de las decisiones de los jueces, y busca principalmente desarrollar el razonamiento lógico jurídico entre los alumnos y la enseñanza de los principios legales que subyacen a dichas decisiones.

Los casos seleccionados para análisis de los estudiantes deben ser importantes o emblemáticos, en el sentido de que normalmente pueden ser demostrativos de una posición jurisprudencial determinada, o porque significaron en un momento histórico un giro en la opinión mayoritaria de la corte o tribunal, o porque abordan a profundidad un tema dogmático que el docente está interesado en que sus alumnos estudien, 
o porque generó gran polémica en los medios judiciales.

Esta metodología, aunque se aplica con bastante frecuencia en las clases de derecho sustantivo, se utiliza en menor grado en la enseñanza del derecho procesal, por cuanto los fallos jurisprudenciales que hacen referencia a las normas de procedimiento son menos frecuentes y tal vez menos emblemáticos de lo que podrían considerarse otro tipo de decisiones judiciales.

Consideramos que el método de casos puede tener utilidad en la enseñanza del derecho procesal, pero especialmente como una metodología complementaria o auxiliar de otras más eficaces y relevantes, como el ABP o los juicios simulados, de los que se habló anteriormente.

\section{La asistencia a juzgados, oficinas de abogados y oficinas estatales}

Desde el punto de vista de la experiencia docente en derecho procesal, podemos afirmar sin lugar a equívocos que invitar a un estudiante a que visite un despacho judicial, asista a una audiencia, acompañe al juez a una diligencia o simplemente observe cómo opera en la práctica cualquier aspecto del procedimiento, así sea solamente la vigilancia judicial de un expediente, resulta una de las estrategias pedagógicas más importantes y más relevantes para efectos de contextualizar los conocimientos que se han estudiado, tanto dentro como fuera del salón de clase, y para que el estudiante asocie con la realidad los conceptos teóricos que ha obtenido con el estudio.
Similar efecto producen las visitas o las pasantías de los estudiantes en las oficinas de abogados o en cualquier dependencia pública. En la medida en que la mayoría de los bufetes de abogados tienen dentro de sus actividades alguna relacionada con el litigio, y considerando también que algunas entidades estatales, así sean del orden administrativo, cumplen funciones jurisdiccionales, el involucramiento y participación directa de un estudiante en el proceso de formulación o de decisión de alguna controversia provoca profundos cambios en la concepción del derecho y, por ende, se constituye en una de las herramientas más valiosas de aqueIlas que puede emplear el docente para lograr el aprendizaje del derecho procesal.

Imaginará el lector de estas líneas que los anteriores comentarios corresponden solamente a la visión de un docente del derecho procesal, y que faltaría entonces la opinión que al respecto emitan los estudiantes que han tenido la oportunidad de vivir esta experiencia. En este sentido quisiera manifestar que, en prácticamente todas las encuestas que se hacen a los estudiantes y en las cuales se pregunta por los aspectos positivos y los aspectos negativos del curso, casi sin excepción resaltan como un aspecto altamente positivo este tipo de experiencias pedagógicas.

Así las cosas, la pregunta que surge es en qué momento del año lectivo o del semestre académico deben programarse dichas visitas o pasantías. Consideramos que la respuesta varía según se trate de simples visitas ocasionales o de pasantías, pues estas últimas tienen una connotación de permanencia en el tiempo que hace recomendable una duración más prolongada. 
Las visitas a despachos judiciales, que por su corta duración se limitan normalmente al transcurso del día, pueden y deben hacerse con frecuencia durante el semestre académico, como un complemento fundamental de cualquier metodología que se emplee en el salón de clase, y procurando que la visita corresponda a la finalización de un módulo importante dentro del programa académico. No resulta aconsejable programarlas cuando el estudiante no se ha adentrado aún en el estudio de los fenómenos que va a observar, pues así podrá malograrse el efecto pedagógico que se persigue con la actividad.

Las visitas a una oficina de abogados implican una duración mayor en el tiempo, dado que los asuntos procesales pueden no ocupar la atención de los abogados de una firma todos los días a toda hora, como sí sucede en un despacho judicial. Así, una periodicidad de unas dos visitas semanales, con una duración de 3 o 4 horas diarias durante un mes calendario, puede perfectamente cumplir el propósito perseguido.

En cuanto a las visitas a oficinas públicas, como pueden serlo las superintendencias, contralorías, procuradurías y otras, estas podrían cumplirse en un mismo día o extenderse en el tiempo como aquellas de las oficinas de abogados, según se considere apropiado a la luz de los propósitos planteados.

Valga anotar que la planeación de estas visitas implica para el docente una labor adicional a la del salón de clase, pues normalmente resulta necesario contactar a los funcionarios del despacho judicial o de la oficina pública, así como también a un abogado del bufete escogido, de manera que la presencia del estudiante pueda programarse en un momento en el que todos los involucrados tengan disponibilidad de tiempo y se vaya a adelantar la actividad práctica para la cual se busca la presencia del estudiante.

Si hay disposición por parte del estudiante, si hay tiempo y voluntad, esta puede ser una de las mejores herramientas al alcance de cualquier docente del derecho procesal. Por tal motivo, no dudamos en recomendarla como una de las más benéficas y positivas.

\section{E. La cátedra magistral}

La denominada "cátedra magistral" tiene una gran tradición en nuestro medio académico coIombiano, pues sin lugar a dudas ha sido uno de los métodos de enseñanza más utilizado en las aulas para la docencia del derecho en general y, por supuesto, también para la pedagogía del derecho procesal.

La cátedra magistral, tal como se emplea en el medio jurídico y como la entienden la mayoría de los docentes, consiste simplemente en la disertación oral por parte del profesor en el salón de clase, y busca transmitir a los alumnos los contenidos dogmáticos de la asignatura de que se trate, junto con los desarrollos doctrinales y jurisprudenciales más recientes, exposición que ocasionalmente viene acompañada de citas anecdóticas y de opiniones o comentarios por parte del docente expositor, que de alguna manera reflejan sus experiencias personales en la vida profesional como abogado. 
Este método de enseñanza ha sido objeto de críticas cada vez más frecuentes por parte de algunos expertos en docencia estudiosos del derecho, porque, por un lado, consideran que la cátedra magistral tiene serias limitaciones al lograr transmitir solamente y de manera imperfecta la dogmática del derecho, pero no desarrolla en el estudiante las habilidades y competencias que algún día lo convertirán en un buen abogado; y por otro lado, consideran que la cátedra magistral contribuye a concentrar la carga principal y la responsabilidad del aprendizaje en cabeza del docente y no del estudiante, que es donde debería estar radicada.

Con relación a las críticas antes señaladas, consideramos conveniente dejar constancia de nuestro disenso en lo que se refiere al manejo del componente teórico que debe incluir toda lección de derecho. Así, parece pertinente rescatar en estas líneas la importancia que en nuestro parecer aún tiene la cátedra magistral para efectos de abordar el aspecto teórico de la disciplina jurídica, especialmente cuando se trata del derecho procesal, la cual permite que el docente ayude al estudiante a integrar los conceptos que ha encontrado dispersos en diferentes fuentes bibliográficas y relacionarlos no solamente entre sí, sino también con la temática del curso.

Algunos autores como Arangüena Fanego (2011, p. 93) comparten la visión de quien escribe estas líneas:

A pesar de las duras críticas de que ha sido objeto el (sic) los últimos tiempos, la lección magistral sigue siendo el método más usado y las razones que explican esta permanencia obedecen sobre todo a su mayor eficacia, en comparación con otros métodos, para la transmisión de información, para la facilitación de la comprensión de temas complejos, para elevar el nivel motivacional de los alumnos, para sintetizar fuentes informativas diversas y de difícil acceso para los estudiantes y por razones de economía y esfuerzo.

Igualmente, Garciandía González \& Liébana Ortiz se expresan en el mismo sentido al manifestar que "De acuerdo con estas ideas, hemos de dejar constancia de la falsedad que esconde, en nuestro criterio, la dialéctica, fomentada últimamente, de contraponer, a modo de panacea o solución a todos los males, las clases teóricas a las clases prácticas, como si a través de las primeras no se pudiera transmitir, junto a la indudable dimensión teórica y abstracta, una enseñanza práctica que le es inseparable" (2011, p. 274).

Toda lección de derecho tiene un componente teórico, dentro del cual deben incluirse no solamente los elementos dogmáticos sino también sus principios y fundamentos, lo cual se puede y debe complementar con opiniones doctrinales y tendencias jurisprudenciales que ayudan a la mejor comprensión y manejo de los temas. Para ese componente teórico, consideramos que la cátedra magistral puede ser de gran ayuda en un proceso pedagógico, el cual será complementado luego por el componente práctico que el docente escoja.

Ahora bien, la cátedra magistral debe tener un elevado contenido participativo por parte de los alumnos, en la medida en que debe permitír- 
seles formular toda clase de preguntas, observaciones, objeciones, críticas o cualquier otro aporte que consideren pertinente para el tema en cuestión, lo cual no solamente contribuye a aclarar dudas conceptuales que puedan tener otros estudiantes, sino que también se constituye en un importantísimo elemento que estimula la atención y participación, y no puede redundar sino en un mayor y mejor aprendizaje.

Así las cosas, pareciera que la cátedra magistral bien concebida y bien empleada puede ser un método eficaz para el manejo del componente teórico de un tema jurídico, y contribuye a la comprensión de la dogmática y de los conceptos que gravitan alrededor de una lección en derecho.

Ahora, merecen comentario también las críticas que se hacen a la cátedra magistral en el sentido de que concentra de manera excesiva la carga del aprendizaje en cabeza del docente, y porque no desarrolla en el alumno competencias y habilidades prácticas, como son su capacidad de investigación, el análisis de problemas y, especialmente, la habilidad de enseñarse a sí mismo.

Resulta cierto que la cátedra magistral puede parecer más cómoda al estudiante como método pedagógico, porque normalmente lo releva de la obligación de preparar las clases y de intervenir activamente en ellas, de manera que su participación en el proceso de aprendizaje puede terminar limitado a una mera presencia física con muchos otros alumnos. Lo anterior parece aún más cierto si dentro del alumnado campea esa actitud demoledora que no dudamos en denominar la de un "consumidor de la educación", la cual consiste en que el estudiante asiste a la clase con la expectativa de que el docente haga todo por él en el proceso de aprendizaje, probablemente fundado en el elevado valor de la matrícula que ha debido pagar, lo cual hace que espere a cambio una retribución equivalente, como si se tratara de un asunto mercantil y no de un proceso pedagógico.

Phillips (2001) por ejemplo, llama la atención sobre este problema de actitud que compromete el aprendizaje, y consiste en que los estudiantes, como consumidores, sienten que no deben preparar la clase sino simplemente asistir para que el docente les enseñe el derecho de una manera eficaz y entretenida. Ellos se convierten, así, en el sujeto pasivo del proceso de aprendizaje, cuando debería ser exactamente lo contrario.

Para evitar que esta situación se presente, resulta recomendable asignar parte importante del proceso pedagógico al estudiante, de manera que al momento de dictar una lección magistral en la clase este haya leído varios autores, haya intentado extraer los conceptos más relevantes de los escritos y haya hecho, al menos, un esfuerzo por relacionarlos entre sí y con el tema de la clase. Lo anterior tendrá la ventaja adicional para el docente, de contar con unos alumnos mejor ilustrados en el asunto de que se trate, lo cual necesariamente elevará el nivel de las intervenciones del estudiante y abonará el terreno para un provechoso aprendizaje.

Por otro lado, debe mencionarse que algunos autores han igualmente descalificado la cátedra magistral como método pedagógico, por cuanto 
estiman que no desarrolla en el alumno las habilidades, destrezas y competencias prácticas que se requieren para un exitoso ejercicio del derecho. Aun cuando la anterior pareciera una aseveración con fundamento, una mirada más cuidadosa al tema indica que si bien no se desarrollan habilidades prácticas, sí se pueden desarrollar habilidades mentales de razonamiento lógico y de manejo de instituciones y conceptos jurídicos que le servirán al alumno en el momento en que se encuentre ante un problema real como abogado.

A lo anterior puede agregarse un argumento que es fruto de la experiencia docente del autor y que, con seguridad, quienes lean estas líneas podrán corroborar, y es que los estudiantes buscan de manera permanente conocer qué posición u opinión tiene el profesor con respecto a uno $u$ otro tema en particular, ya sea para discutirlo y descartarlo o para que les sirva de guía, pero en todo caso sienten que es labor del docente expresar de manera inequívoca su opinión con respecto al asunto que ocupa la atención de la clase en un momento determinado. Tal vez esta situación se explique por cuanto el alumno no ha adquirido aún la suficiente seguridad en sí mismo como abogado, y busca una guía inicial por parte del docente para que sea este quien, a través de argumentos debidamente sustentados, vaya guiándolo y ayudándolo a construir su propio criterio jurídico.

Saiz Garitaonandia resume la importancia de la cátedra magistral cuando afirma que

Es difícil considerar un método que prescinda de forma absoluta de esta fórmula, por lo que nosotros apostamos por una metodología mixta que vincule la teoría y la práctica de forma tal que una no pueda entenderse sin la otra. Además, debemos considerar que, en general, la implementación de los estudios de grado está acarreando una reducción de las horas lectivas de cada asignatura, por lo que la capacidad que tengamos de maximizar dichas horas será fundamental para que los estudiantes puedan aprehender las competencias que sobre la misma queremos transmitirles. (2011, p. 391).

En síntesis, si bien no sea razonable afirmar que la cátedra magistral pueda constituirse en el eje o soporte fundamental de una metodología para la enseñanza de temas de naturaleza procesal, sí parece posible sostener que no ha perdido aún su vigencia como elemento docente de gran importancia en el salón de clase, especialmente cuando se trata de abordar la parte teórica de los temas que posteriormente se trabajarán de manera práctica a través de casos, talleres, tribunales simulados y otros.

\section{F. Otras alternativas pedagógicas}

Finalmente, quisiéramos hacer referencia a algunas alternativas adicionales que se encuentran a disposición del docente de derecho procesal, todas encaminadas a reforzar el componente práctico de la clase, y a las cuales puede recurrir para ilustrar, contextualizar o profundizar en un tema cualquiera.

La primera de ellas se refiere a la proyección en el salón de clase de videos de audiencias o diligencias procesales. En la medida en que la regla técnica de la oralidad ha ido ganando te- 
rreno por la celeridad que imprime a los procesos judiciales, resulta cada vez más fácil tener acceso a dichas reproducciones, que pueden remplazar en alguna medida la experiencia de haber asistido personalmente a ellas.

Naturalmente, luego de la proyección de un video debe seguir una sesión de discusión en clase, con la participación de todos los estudiantes, en la cual el docente guíe el análisis hacia los aspectos legales que considere más relevantes, procurando siempre asociarlos con el tema de la clase en general y con los conceptos cuya aplicación práctica pretende ilustrar con la actividad. El valor pedagógico de esta alternativa se concentra principalmente en la discusión que se genera luego de la proyección del video, pues se aclaran las dudas, los conceptos que se manejaron, se destaca lo bueno y lo malo de la actuación de cada una de las partes, y se hacen recomendaciones y sugerencias que pueden ser de gran valor en la vida profesional de un abogado.

La segunda actividad consiste en la redacción de memoriales y escritos procesales por parte de los estudiantes, lo cual debe hacerse con fundamento en un caso o una situación problemática que haya diseñado previamente el profesor y entregado a los alumnos, y en virtud del cual el estudiante asuma el rol de una de las partes en el proceso y defienda una posición determinada ante una decisión judicial adversa o por la urgencia de una petición que resulta indispensable. Esta actividad fomenta las habilidades de argumentación y de expresión escrita del alumno, y le proporciona una oportunidad de poner en práctica los conceptos aprendidos, relacionándolos lógicamente con los hechos de un caso.
La tercera actividad pedagógica práctica consiste en los denominados trabajos en grupo, los cuales pueden hacerse, tanto dentro del salón de clase como por fuera de él. Una de las habilidades que resultan fundamentales en los abogados es la capacidad de trabajar en equipo, y esta práctica les brinda la oportunidad perfecta para desarrollar o perfeccionar dicha destreza.

Sin embargo, la experiencia muestra que, como regla general, resulta más productiva la actividad y resultan más auténticos los trabajos que se hacen dentro del salón de clase que por fuera de él, en la medida en que el docente puede estar monitoreando cuáles miembros del grupo se encuentran trabajando en ella y cuáles están haciendo cualquier otra cosa diferente.

Otra manera de controlar que todos los miembros del equipo hayan contribuido al trabajo elaborado, estrategia que es utilizada con alguna frecuencia por los profesores, es programar una exposición oral en la que el docente se reserve la facultad de escoger cuál de los integrantes del grupo hará la presentación. La mera perspectiva de ser el orador escogido hace que los estudiantes se involucren más en la actividad en grupo, y aunque la motivación así generada deja mucho que desear, sí resulta positivo el resultado que con esta estrategia se obtiene.

\section{CONCLUSIONES}

Hemos visto en el presente escrito que existe una tendencia clara y marcada para la enseñanza en general, de trasladar la responsabilidad y la carga docente del profesor hacia el estudiante. 
De la misma manera, existe una tendencia que busca empoderar al alumno para que desarroIle las habilidades que resultan necesarias para procurarse su propio aprendizaje. Se quiere que el estudiante aprenda a aprender por sí solo.

Por otro lado, hemos visto que en la enseñanza jurídica, y concretamente en la enseñanza del derecho procesal, esa tendencia hacia actividades prácticas que desarrollen en el estudiante las habilidades que algún día necesitará como abogado en ejercicio, es más marcada todavía. Actualmente, no es posible concebir el manejo de un curso de derecho procesal con el empleo de metodologías de naturaleza teórica, pues el carácter instrumental del derecho procesal con respecto al derecho sustantivo impone el empleo de actividades prácticas que acerquen al estudiante a la realidad del ejercicio profesional.

Para concretar una metodología práctica en la enseñanza del derecho procesal resultan especialmente recomendables metodologías tales como el aprendizaje basado en problemas (ABP), el método de casos, el empleo de juicios simulados, las visitas o pasantías a los despachos judiciales y a las oficinas de abogados, sin perjuicio de otras actividades complementarias que pueden utilizarse igualmente por parte del docente.

Sabemos, también, que el rol del profesor en el salón de clase resulta absolutamente determinante para la motivación y el aprendizaje del estudiante, y que ya contamos con un interés suficiente de los alumnos por el derecho procesal.

No obstante lo anterior, el éxito que puedan tener estas estrategias o metodologías prácticas depende en buena parte del empeño que ponga el estudiante y del trabajo y tiempo que decida dedicar a su propio aprendizaje. Una de las grandes amenazas que se ciernen sobre esta tendencia pedagógica práctica es la costumbre, bien arraigada del estudiante, de pretender que el docente le entregue todo listo para su digestión y asimilación, y la consecuente resistencia a asumir un papel protagónico en su propio proceso de aprendizaje.

Otra amenaza que resulta casi más intimidante es el factor tiempo. La cantidad y la complejidad de los temas que conforman el derecho procesal, conducen a que se requiera de un tiempo considerable para el estudio de cada institución jurídica, aunque hay unas más elementales que otras, como es natural. Así, un semestre académico o un año lectivo, descontadas las vacaciones, los festivos y los días en que por cualquier motivo no es posible adelantar la clase, da como resultado un tiempo insuficiente para evacuarlos satisfactoriamente, más aún si se intenta hacerlo a través de numerosas actividades prácticas que consumen buena parte del que hay disponible.

Así las cosas, no parece viable reducir los temas del programa de derecho procesal, pues una vez revisados puede advertirse que todos resultan fundamentales y sería un craso error prescindir de cualquiera de ellos. Igualmente, si se intenta incrementar el trabajo del alumno por fuera de clase, llegará en algún momento el reclamo del estudiante por la falta de concordancia entre el número de créditos académicos que le reconoce la materia versus el número de horas que debe dedicar a su estudio, y debe aceptarse que algo 
de razón le asiste. Así, tampoco parece viable adicionar créditos al estudio del derecho procesal ni alargar el tiempo disponible en el pénsum para estudiarlo.

Quedará, entonces, en cabeza del docente y de sus alumnos lograr maximizar de la mejor manera posible el tiempo disponible, y hacer votos porque una mixtura de metodologías prácticas y teóricas, junto con actividades complementarias, logre el cometido de mejorar la comprensión y asimilación de los conceptos del derecho procesal por parte de los estudiantes.

\section{Bibliografía}

Anderson, L. C., \& Kirkwood, C. E. (1987). Teaching Civil Procedure With The Aid of Local Tort Litigation. Journal of Legal Education, 215-231.

Arangüena Fanego, C. (2011). Las nuevas tecnologías aplicadas a la docencia del derecho procesal. En J. Picó I Junoy, El aprendizaje del derecho procesal (pág. 93). Barcelona: Bosch.

Arnon, S. \& Reichel, N. (2007). Who is the ideal teacher? Am I? Similarity and difference in perception of students of education regarding the qualities of a good teacher and of their own qualities as teachers. Teachers and Teaching: theory and practice, 441-464.

Caballol Angelats, L. (2011). La docencia del derecho procesal basada en problemas. En J. Picó I Junoy, El aprendizaje del derecho procesal (pág. 145). Barcelona: Bosch.
Frank, J. (1933). What Constitutes a Good Legal Education? American Bar Association Journal, 723-728.

Garciandía González, P. M. \& Liébana Ortiz, J. R. (2011). La “Nueva” enseñanza del derecho procesal (II): aproximación a la metodología docente a través de varias propuestas prácticas. En J. Picó I Junoy, El aprendizaje del derecho procesal. Nuevos retos de la enseñanza universitaria (pág. 274). Barcelona: Bosch.

Phillips, J. J. (2001). Law School Teaching. Saint Louis Unversity Law Journal, 725-730.

Rivkin, S. G., Hanushek, E. A. \& Kain, J. F. (2005). Teachers, Schools, and Academic Achievement. Econometrica, 417-458.

Saiz Garitaonandia, A. (2011). Uso de múltiples medidas para incentivar la motivación en el aprendizaje del derecho procesal penal. En J. Picó I Junoy, El aprendizaje del derecho procesal. Nuevos retos de la enseñanza universitaria. Barcelona: Bosch.

SchneIDER, E. N. (1987). Rethinking the Teaching of Civil Procedure. Journal of Legal Education, 41-45.

ShapiRo, S. J. (2000-2001). Teaching First-Year Civil Procedure and Other Introductory Courses by the Problem Method. Creighton Law Review, 245-282.

Sheinkopf Hoffman, L. (2003). A Parting Reprise (Teaching Civil Procedure). Saint Louis University Law Journal, 43-57. 
Subrin, S. N. (1993-1994). Teaching Civil Procedure While You Watch It Disintegrate. Brooklyn Law Review, 1155-1190.

SzkLARSKI, A. (2011). Pupils' Experience of Being Motivated to Learn in School: An Empirical Phenomenological Study. Teaching Science, 43-47.

TAN, S. (2008-2009). Perceptions of Students on Factors in Motivation to Learn. E-Journal of New World Sciences Academy, 155-167.

Teply, L. L. \& Whitten, R. U. (2003). Teaching Civil Procedure Using an Integrated Case-Text-and-
Problem Method. Saint Louis University Law Journal, 91-110.

The Princeton Review. (s.f.). Recuperado el 13 de agosto de 2012, de http://www.princetonreview.com/law/case-method.aspx

Wheaton, C. C. (1936-1937). Law Teaching and Pragmatism. Georgetown Law Journal, 338350.

WiLdman, C. E. (1942). The Teacher Who Is Remembered. The Modern Language Journal, 564-572. 